\title{
Simulation of Bipolar Charge Transport in Graphene by Using a Discontinuous Galerkin Method
}

\author{
Armando Majorana ${ }^{1}$, Giovanni Nastasi ${ }^{1}$ and Vittorio Romano ${ }^{1, *}$ \\ ${ }^{1}$ Department of Mathematics and Computer Science, Università degli Studi di \\ Catania, viale Andrea Doria 6, 95125 Catania, Italy.
}

Received 23 February 2018; Accepted (in revised version) 9 July 2018

\begin{abstract}
Charge transport in suspended monolayer graphene is simulated by a numerical deterministic approach, based on a discontinuous Galerkin (DG) method, for solving the semiclassical Boltzmann equation for electrons. Both the conduction and valence bands are included and the interband scatterings are taken into account.

The use of a Direct Simulation Monte Carlo (DSMC) approach, which properly describes the interband scatterings, is computationally very expensive because the valence band is very populated and a huge number of particles is needed. Also the choice of simulating holes instead of electrons does not overcome the problem because there is a certain degree of ambiguity in the generation and recombination terms of electronhole pairs. Often, direct solutions of the Boltzmann equations with a DSMC neglect the interband scatterings on the basis of physical arguments. The DG approach does not suffer from the previous drawbacks and requires a reasonable computing effort.

In the present paper the importance of the interband scatterings is accurately evaluated for several values of the Fermi energy, addressing the issue related to the validity of neglecting the generation-recombination terms. It is found out that the inclusion of the interband scatterings produces huge variations in the average values, as the current, with zero Fermi energy while, as expected, the effect of the interband scattering becomes negligible by increasing the absolute value of the Fermi energy.
\end{abstract}

AMS subject classifications: 82D37, 82C70, 65M60, 82C80

Key words: Graphene, bipolar charge transport, discontinuous Galerkin method.

\section{Introduction}

The last years have witnessed a great interest in 2D-materials for their promising applications. The most investigated one is graphene which is considered as a potential

*Corresponding author. Email addresses: majorana@dmi.unict.it (A. Majorana), g.nastasi@unict.it (G. Nastasi), romano@dmi. unict.it (V. Romano) 
new semiconductor material for future applications in nano-electronic [1-4] and optoelectronic devices [5]. A reasonable and physically accurate model for charge transport is based on semiclassical Boltzmann equations (quantum effects have also been included in the literature, e.g. see [6,7]). Usually, the available solutions have been obtained by direct Monte Carlo simulations, e.g. a new Direct Simulation Monte Carlo (DSMC) procedure has been devised in [8-10] in order to properly take into account the Pauli exclusion principle. Direct solutions of the electron transport equations with finite difference methods have been obtained in [3] while a Discontinuous Galerkin (DG) method has been used in $[8,11,12]$. See $[13,14]$ for application of the DG method to traditional semiconductors, while numerical schemes for the Wigner equation can be found in [15]. A hydrodynamical model based on the maximum entropy principle (MEP) has been formulated in [16] using a set of field variables which proved to be successful for traditional semiconductors as silicon [17-23], gallium arsenide [17,24], silicon carbide [25]. In general both electrons in the conduction and valence bands contribute to charge transport in graphene and the zero gap energy band allows for the creation of electron-hole pairs by scattering with phonons around the Dirac points. Therefore, one has also to include interband electronphonon scatterings. However, if a gate voltage is applied, it is possible to modify the value of the Fermi energy $\varepsilon_{F}$ creating a kind of doping as in conventional semiconductors. If $\varepsilon_{F}$ is positive and high enough, one has a kind of $n$-doping and the only relevant contribution to the current is due to the electrons in the conduction band. Analogously, if $\varepsilon_{F}<0$ one has a kind of $p$-doping. The use of DSMC in the bipolar case is rather heavy from a computational point of view because the valence band is very populated and a huge number of simulation particles is needed. A viable way to overcome the problem could be to simulate, in the valence band, holes instead of electrons. Unfortunately, this introduces a certain degree of ambiguity in the generation and recombination terms of electron-hole pairs and makes the approach rather questionable, as explained in the next section. For such a reason, often the interband scattering is neglected. The DG method does not suffer from the previous difficulties and keeps the computational effort to a reasonable level. In the present paper, by performing an extensive numerical simulation with the DG method of the system of Boltzmann equations for electrons in the conduction and valence bands, the importance of the interband scatterings is accurately evaluated for several values of the Fermi energy in the case of suspended monolayer graphene under a constant external electric field. It is addressed the issue related to the validity of neglecting the generation-recombination terms. It is found out that the inclusion of the interband scatterings induces huge variations in the average values, as the current, with zero Fermi energy while, as expected, the effect of the interband scatterings becomes negligible by increasing in absolute value the Fermi energy. The plan of the paper is as follows. In section 2 the semiclassical model of charge transport in graphene is recalled. In particular, there are highlighted the problems arising in the electron-hole scatterings. In section 3 the DG method, adopted in the paper, is explained. At last, in section 4 the numerical results are presented and the issue of the role played by the interband scatterings is analyzed. Some details are postponed to the Appendix. 


\section{The semiclassical charge transport in graphene}

In a semiclassical kinetic setting, the charge transport in graphene is described, in general, by four Boltzmann equations, one for electrons in the valence $(\pi)$ band and one for electrons in the conduction $\left(\pi^{*}\right)$ band, that in turn can belong to the $K$ or $K^{\prime}$ valley. By assuming the $K$ and $K^{\prime}$ valleys as equivalent, then we can consider only the two equations

$$
\frac{\partial f_{s}}{\partial \mathrm{t}}+\mathbf{v}_{s} \cdot \nabla_{\mathbf{x}} f_{s}-\frac{e}{\hbar} \mathbf{E} \cdot \nabla_{\mathbf{k}} f_{s}=Q\left(f_{s}, f_{-s}\right) \quad(s= \pm 1),
$$

where $f_{s}=f_{s}(\mathbf{t}, \mathbf{x}, \mathbf{k})$ represents the distribution function of charge carriers in the conduction band $(\mathrm{CB})$, for $s=1$, or in the valence band (VB), for $s=-1$, at position $\mathrm{x}$, time $t$ and wave-vector $\mathbf{k}$. We denote by $\nabla_{\mathbf{x}}$ and $\nabla_{\mathbf{k}}$ the gradients with respect to the position and wave vector, respectively. In the sequel, for simplifying the notation, we write also $f_{+}$ and $f_{-}$to indicate the distributions of electrons in the conduction and valence bands.

The group velocity $\mathbf{v}_{s}$ is related to the energy band $\varepsilon_{s}$ by

$$
\mathbf{v}_{s}=\frac{1}{\hbar} \nabla_{\mathbf{k}} \varepsilon_{S} .
$$

With a very good approximation [2] a linear dispersion relation holds for the energy bands $\varepsilon_{s}$ around the Dirac points; so that, choosing the origin of the reference frame in the $\mathbf{k}$-space coinciding with a Dirac point, we have $\varepsilon_{s}=s \hbar v_{F}|\mathbf{k}|$, where $v_{F}$ is the (constant) Fermi velocity and $\hbar$ the Planck constant divided by $2 \pi$. The elementary (positive) charge is denoted by $e$. Here the electric field $\mathbf{E}$ is assumed as external, and therefore we do not include the Poisson equation. The right hand side of Eq. (2.1) is the collision term representing the interaction of electrons with acoustic, optical and $K$ phonons. Acoustic phonon scattering is intravalley and intraband. Optical phonon scattering is intravalley and can be both longitudinal (LO) and transversal (TO). It can be intraband, that is it leaves electrons in the same band, or interband pushing electrons from an initial band to the other one. Scattering with optical phonon of type $K$ pushes electrons from a valley to a neighbor one (intervalley scattering). We assume that phonons are at thermal equilibrium. Hence, the general form of the collision term can be written as

$$
\begin{aligned}
Q\left(f_{s}, f_{-s}\right)= & \sum_{s^{\prime}}\left[\int_{\mathbb{R}^{2}} S_{s^{\prime}, s}\left(\mathbf{k}^{\prime}, \mathbf{k}\right) f_{s^{\prime}}\left(\mathbf{t}, \mathbf{x}, \mathbf{k}^{\prime}\right)\left(1-f_{s}(\mathbf{t}, \mathbf{x}, \mathbf{k})\right) d \mathbf{k}^{\prime}\right. \\
& \left.-\int_{\mathbb{R}^{2}} S_{s, s^{\prime}}\left(\mathbf{k}, \mathbf{k}^{\prime}\right) f_{s}(\mathbf{t}, \mathbf{x}, \mathbf{k})\left(1-f_{s^{\prime}}\left(\mathbf{t}, \mathbf{x}, \mathbf{k}^{\prime}\right)\right) d \mathbf{k}^{\prime}\right],
\end{aligned}
$$

where the total transition rate is given by the sum of the contributions of the several types of scatterings

$$
\begin{aligned}
S_{S^{\prime}, S}\left(\mathbf{k}^{\prime}, \mathbf{k}\right)=\sum_{v}\left|G_{S^{\prime}, S}^{(v)}\left(\mathbf{k}^{\prime}, \mathbf{k}\right)\right|^{2}[ & \left(n_{\mathbf{q}}^{(v)}+1\right) \delta\left(\varepsilon_{S}(\mathbf{k})-\varepsilon_{\mathcal{S}^{\prime}}\left(\mathbf{k}^{\prime}\right)+\hbar \omega_{\mathbf{q}}^{(v)}\right) \\
& \left.+n_{\mathbf{q}}^{(v)} \delta\left(\varepsilon_{S}(\mathbf{k})-\varepsilon_{S^{\prime}}\left(\mathbf{k}^{\prime}\right)-\hbar \omega_{\mathbf{q}}^{(v)}\right)\right] .
\end{aligned}
$$


The index $v$ labels the $v$ th phonon mode, $\left|G_{s^{\prime}, S}^{(v)}\left(\mathbf{k}^{\prime}, \mathbf{k}\right)\right|$ is the matrix element, which describes the scattering mechanism, due to phonons of type $v$, between electrons belonging to the band $s^{\prime}$ and electrons belonging to the band $s$. The symbol $\delta$ denotes the Dirac distribution function, $\omega_{\mathbf{q}}^{(v)}$ is the the $v$ th phonon frequency, $n_{\mathbf{q}}^{(v)}$ is the Bose-Einstein distribution for the phonon of type $v$

$$
n_{\mathbf{q}}^{(v)}=\frac{1}{e^{\hbar \omega_{\mathbf{q}}^{(v)} / k_{B} T}-1},
$$

$k_{B}$ is the Boltzmann constant and $T$ is the graphene lattice temperature which, in this article, will be kept constant. When, for a phonon $v_{*}, \hbar \omega_{\mathbf{q}}^{\left(v_{*}\right)} \ll k_{B} T$, then the scattering with the phonon $v_{*}$ can be assumed elastic. In this case, we eliminate in Eq. (2.2) the term $\hbar \omega_{\mathbf{q}}^{\left(v_{*}\right)}$ inside the delta distribution and we use the approximation $n_{\mathbf{q}}^{\left(v_{*}\right)}+1 \approx n_{\mathbf{q}}^{\left(v_{*}\right)}$.

Now we write explicitly the transition rates used in our simulations.

For acoustic phonons, usually one considers the elastic approximation, and

$$
2 n_{\mathbf{q}}^{(a c)}\left|G^{(a c)}\left(\mathbf{k}^{\prime}, \mathbf{k}\right)\right|^{2}=\frac{1}{(2 \pi)^{2}} \frac{\pi D_{a c}^{2} k_{B} T}{2 \hbar \sigma_{m} v_{p}^{2}}\left(1+\cos \vartheta_{\mathbf{k}, \mathbf{k}^{\prime}}\right)
$$

where $D_{a c}$ is the acoustic phonon coupling constant, $v_{p}$ is the sound speed in graphene, $\sigma_{m}$ the graphene areal density, and $\vartheta_{\mathbf{k}, \mathbf{k}^{\prime}}$ is the convex angle between $\mathbf{k}$ and $\mathbf{k}^{\prime}$.

There are three relevant optical phonon scatterings: the longitudinal optical (LO), the transversal optical (TO) and the $K$ phonons. The matrix elements are

$$
\begin{aligned}
& \left|G^{(L O)}\left(\mathbf{k}^{\prime}, \mathbf{k}\right)\right|^{2}+\left|G^{(T O)}\left(\mathbf{k}^{\prime}, \mathbf{k}\right)\right|^{2}=\frac{2}{(2 \pi)^{2}} \frac{\pi D_{O}^{2}}{\sigma_{m} \omega_{O}}, \\
& \left|G^{(K)}\left(\mathbf{k}^{\prime}, \mathbf{k}\right)\right|^{2}=\frac{1}{(2 \pi)^{2}} \frac{2 \pi D_{K}^{2}}{\sigma_{m} \omega_{K}}\left(1-\cos \vartheta_{\mathbf{k}, \mathbf{k}^{\prime}}\right),
\end{aligned}
$$

where $D_{O}$ is the optical phonon coupling constant, $\omega_{O}$ the optical phonon frequency, $D_{K}$ is the K-phonon coupling constant and $\omega_{K}$ the K-phonon frequency. Physical parameters for the collision terms are summarized in Table 1.

Table 1: Physical parameters for the collision terms.

\begin{tabular}{||l|c||l|c||}
\hline$v_{F}$ & $10^{8} \mathrm{~cm} / \mathrm{s}$ & $v_{p}$ & $2 \times 10^{6} \mathrm{~cm} / \mathrm{s}$ \\
\hline$\sigma_{m}$ & $7.6 \times 10^{-8} \mathrm{~g} / \mathrm{cm}^{2}$ & $D_{a c}$ & $6.8 \mathrm{eV}$ \\
\hline$\hbar \omega_{O}$ & $164.6 \mathrm{meV}$ & $D_{O}$ & $10^{9} \mathrm{eV} / \mathrm{cm}$ \\
\hline$\hbar \omega_{K}$ & $124 \mathrm{meV}$ & $D_{K}$ & $3.5 \times 10^{8} \mathrm{eV} / \mathrm{cm}$ \\
\hline
\end{tabular}

In the valence band, instead of electrons, it is possible to consider holes. If we indicate with $\mathbf{k}_{e}$ the state of a missing electron in the VB and with $\mathbf{k}_{h}$ and $\varepsilon_{h}\left(\mathbf{k}_{h}\right)$ the state and the 
energy of the corresponding hole, they are related by the conditions $[26,27]$

$$
\mathbf{k}_{h}=-\mathbf{k}_{e}, \quad \varepsilon_{h}\left(\mathbf{k}_{h}\right)=-\varepsilon_{-}\left(\mathbf{k}_{e}\right) .
$$

Removing the labels to avoid unnecessarily complicated notation, the dispersion relation for the energy of holes writes

$$
\varepsilon_{h}(\mathbf{k})=\hbar v_{F}|\mathbf{k}| .
$$

In some classical textbooks a different formulation of the theory of holes is proposed. In this paper we prefer the approach described in $[26,27]$ because, in our opinion, is more clear from a physical point of view.

Introducing the distribution function of holes in the VB by

$$
f_{h}(\mathbf{t}, \mathbf{x}, \mathbf{k})=1-f_{-}(\mathbf{t}, \mathbf{x},-\mathbf{k}),
$$

after some algebra (the details are postponed to the Appendix A) we obtain that $f_{h}$ satisfies the equation

$$
\partial_{t} f_{h}(\mathbf{t}, \mathbf{x}, \mathbf{k})+\mathbf{v}_{h}(\mathbf{k}) \cdot \nabla_{\mathbf{x}} f_{h}(\mathbf{t}, \mathbf{x}, \mathbf{k})+\frac{e}{\hbar} \mathbf{E} \cdot \nabla_{\mathbf{k}} f_{h}(\mathbf{t}, \mathbf{x}, \mathbf{k})=Q_{h}\left(f_{h}, f_{+}\right)(\mathbf{t}, \mathbf{x}, \mathbf{k}),
$$

where $\mathbf{v}_{h}$ is the group velocity of holes, that it can be shown to take the expression

$$
\mathbf{v}_{h}(\mathbf{k})=\frac{1}{\hbar} \nabla_{\mathbf{k}} \varepsilon_{h}(\mathbf{k}),
$$

and $Q_{h}$ is the collisional term for holes, which splits into

$$
Q_{h}\left(f_{h}, f_{+}\right)=Q_{h h}\left(f_{h}, f_{h}\right)+Q_{h e}\left(f_{h}, f_{+}\right) .
$$

Here (by omitting the explicit dependence on $t$ and $\mathbf{x}$ )

$$
Q_{h h}=-\int_{\mathbb{R}^{2}}\left[S_{-,-}\left(-\mathbf{k}^{\prime},-\mathbf{k}\right) f_{h}(\mathbf{k})\left(1-f_{h}\left(\mathbf{k}^{\prime}\right)\right)-S_{-,-}\left(-\mathbf{k},-\mathbf{k}^{\prime}\right) f_{h}\left(\mathbf{k}^{\prime}\right)\left(1-f_{h}(\mathbf{k})\right)\right] d \mathbf{k}^{\prime}
$$

gives the intraband hole interactions (with phonons), while

$$
Q_{h e}=-\int_{\mathbb{R}^{2}}\left[S_{+,-}\left(\mathbf{k}^{\prime},-\mathbf{k}\right) f_{+}\left(\mathbf{k}^{\prime}\right) f_{h}(\mathbf{k})-S_{-,+}\left(-\mathbf{k}, \mathbf{k}^{\prime}\right)\left(1-f_{h}(\mathbf{k})\right)\left(1-f_{+}\left(\mathbf{k}^{\prime}\right)\right)\right] d \mathbf{k}^{\prime}
$$

represents the interband scatterings (with phonons).

At the equilibrium the distribution function of electrons in both bands is the FermiDirac distribution, given by

$$
f_{F D}^{ \pm}(\mathbf{t}, \mathbf{x}, \mathbf{k})=\frac{1}{1+\exp \left(\frac{\varepsilon_{ \pm}-\varepsilon_{F}}{k_{B} T}\right)},
$$


where $\varepsilon_{F}$ is the Fermi energy and the sign \pm indicates the $\mathrm{CB}(+)$ or the VB $(-)$. Therefore, the equilibrium distribution of holes is given by

$$
f_{F D}^{h}(\mathbf{t}, \mathbf{x}, \mathbf{k})=1-f_{F D}^{-}(\mathbf{t}, \mathbf{x},-\mathbf{k})=1-\frac{1}{1+\exp \left(\frac{\varepsilon_{-}-\varepsilon_{F}}{k_{B} T}\right)}=\frac{1}{1+\exp \left(\frac{\varepsilon_{h}+\varepsilon_{F}}{k_{B} T}\right)}, \quad \varepsilon_{h}>0,
$$

that goes to 0 when $\varepsilon_{h} \mapsto+\infty$. We remark that, instead, $f_{F D}^{-} \mapsto 1$ when $\varepsilon_{-} \mapsto-\infty$.

If we take into account only intraband transitions, the equations are uncoupled, that is each particle belongs to the same population during the simulation. On the contrary, if we have also interband transitions it can happen for a particle to change energy band and thus population.

In the first case a DSMC algorithm can be construct by solving two Boltzmann equations: one for electrons in $\mathrm{CB}$ and one for holes in VB. In particular, the following relations

$$
S_{-,-}\left(-\mathbf{k}^{\prime},-\mathbf{k}\right)=S_{-,-}\left(\mathbf{k}^{\prime}, \mathbf{k}\right)=S_{+,+}\left(\mathbf{k}, \mathbf{k}^{\prime}\right)
$$

hold.

Thus, the Boltzmann equation for holes in $\mathrm{VB}$, in the case when only intraband transitions occur, is the same of that of electrons in $C B$, except for the sign in front of the elementary charge.

\section{The numerical method}

Since we expect an exponential decay of $f_{+}$and $1-f_{-}$, as $|\mathbf{k}| \rightarrow+\infty$, it is reasonable to choose a compact domain $\Omega \subseteq \mathbb{R}^{2}$, such that $f_{+}(t, \mathbf{x}, \mathbf{k}) \approx 0$ and $1-f_{-}(t, \mathbf{x}, \mathbf{k}) \approx 0$, for every $\mathbf{k} \notin \Omega$, and for every $\mathbf{x}$ and $t>0$.

Now we introduce a finite decomposition $\left\{C_{\alpha}: \alpha=1,2, \cdots, N\right\}$ of the domain $\Omega$, with the $C_{\alpha}$ 's open sets such that

$$
C_{\alpha} \subseteq \Omega \quad \forall \alpha, \quad C_{\alpha} \cap C_{\beta}=\varnothing \quad \forall \alpha \neq \beta, \quad \bigcup_{\alpha=1}^{N} \bar{C}_{\alpha}=\Omega .
$$

We assume a constant approximation for each distribution function $f_{s}$ in every cell $C_{\alpha}$. If we denote by $\chi_{\alpha}$ the characteristic function relative to the cell $C_{\alpha}$, then

$$
f_{s}(\mathbf{t}, \mathbf{x}, \mathbf{k}) \approx f_{s}^{\alpha}(\mathrm{t}, \mathbf{x}), \forall \mathbf{k} \in C_{\alpha} \Longleftrightarrow f_{s}(\mathrm{t}, \mathbf{x}, \mathbf{k}) \approx \sum_{\alpha=1}^{N} f_{s}^{\alpha}(\mathrm{t}, \mathbf{x}) \chi_{\alpha}(\mathbf{k}), \forall \mathbf{k} \in \bigcup_{\alpha=1}^{N} C_{\alpha} .
$$

A set of partial differential equations is now derived from the Boltzmann equations (2.1). The new unknowns are the $f_{s}^{\alpha}(\mathbf{t}, \mathbf{x})^{\prime}$ 's instead of the distribution functions $f_{s}(\mathbf{t}, \mathbf{x}, \mathbf{k})$. Formally integrating Eq. (2.1) over each cell $C_{\alpha}$, we obtain

$$
\int_{C_{\alpha}} \frac{\partial f_{s}}{\partial \mathrm{t}} d \mathbf{k}+\int_{C_{\alpha}} \mathbf{v}_{s} \cdot \nabla_{\mathbf{x}} f_{s} d \mathbf{k}-\int_{C_{\alpha}} \frac{e}{\hbar} \mathbf{E} \cdot \nabla_{\mathbf{k}} f_{s} d \mathbf{k}=\int_{C_{\alpha}} Q\left(f_{s}, f_{-s}\right) d \mathbf{k} .
$$


The first two integrals of Eq. (3.1) can be approximated easily; in fact we have

$$
\int_{C_{\alpha}} \frac{\partial f_{s}}{\partial \mathrm{t}}(\mathrm{t}, \mathbf{x}, \mathbf{k}) d \mathbf{k}+\int_{C_{\alpha}} \mathbf{v}_{s} \cdot \nabla_{\mathbf{x}} f_{s}(\mathbf{t}, \mathbf{x}, \mathbf{k}) d \mathbf{k} \approx \operatorname{meas}\left(C_{\alpha}\right) \frac{\partial f_{s}^{\alpha}}{\partial \mathrm{t}}(\mathbf{t}, \mathbf{x})+\left[\int_{C_{\alpha}} \mathbf{v}_{s} d \mathbf{k}\right] \cdot \nabla_{\mathbf{x}} f_{s}^{\alpha}(\mathbf{t}, \mathbf{x}),
$$

where meas $\left(C_{\alpha}\right)$ is the measure (area) of the cell $C_{\alpha}$.

The integral involving the electric field is transformed by using the Gauss theorem. Of course, the result is strictly related to the geometry of the cells. We have used a reconstruction of the fluxes based on a Min-Mod slope limiter. The interested reader is refereed to [9] for all the details about the treatment of the drift term.

The spatial gradient $\nabla_{\mathbf{x}} f_{s}(\mathbf{t}, \mathbf{x}, \mathbf{k})$ can be discretized in the same way as the drift one. In this case the discretization depends on the geometry of the spatial domain and boundary conditions (see for instance $[13,14])$. Since in this paper we consider only space homogeneous solutions, the details are skipped.

The treatment of the term arising from the collision operator is straightforward. If $\mathbf{k} \in C_{\alpha}$, then we have

$$
\begin{aligned}
Q\left(f_{s}, f_{-s}\right)= & \sum_{s^{\prime}}\left[\left(1-f_{s}(\mathbf{t}, \mathbf{x}, \mathbf{k})\right) \int S_{s^{\prime}, s}\left(\mathbf{k}^{\prime}, \mathbf{k}\right) f_{s^{\prime}}\left(\mathbf{t}, \mathbf{x}, \mathbf{k}^{\prime}\right) d \mathbf{k}^{\prime}\right. \\
& \left.-f_{s}(\mathbf{t}, \mathbf{x}, \mathbf{k}) \int S_{s, s^{\prime}}\left(\mathbf{k}, \mathbf{k}^{\prime}\right)\left(1-f_{s^{\prime}}\left(\mathbf{t}, \mathbf{x}, \mathbf{k}^{\prime}\right)\right) d \mathbf{k}^{\prime}\right] \\
\approx & \sum_{s^{\prime}} \sum_{\beta=1}^{N}\left[\left(1-f_{s}^{\alpha}(\mathbf{t}, \mathbf{x})\right) \int_{C_{\beta}} S_{s^{\prime}, s}\left(\mathbf{k}^{\prime}, \mathbf{k}\right) f_{s^{\prime}}\left(\mathbf{t}, \mathbf{x}, \mathbf{k}^{\prime}\right) d \mathbf{k}^{\prime}\right. \\
& \left.-f_{s}^{\alpha}(\mathbf{t}, \mathbf{x}) \int_{C_{\beta}} S_{s, s^{\prime}}\left(\mathbf{k}, \mathbf{k}^{\prime}\right)\left(1-f_{s^{\prime}}\left(\mathbf{t}, \mathbf{x}, \mathbf{k}^{\prime}\right)\right) d \mathbf{k}^{\prime}\right] \\
\approx & \sum_{s^{\prime}} \sum_{\beta=1}^{N}\left[\left(1-f_{s}^{\alpha}(\mathbf{t}, \mathbf{x})\right) f_{s^{\prime}}^{\beta}(\mathbf{t}, \mathbf{x}) \int_{C_{\beta}} S_{s^{\prime}, s}\left(\mathbf{k}^{\prime}, \mathbf{k}\right) d \mathbf{k}^{\prime}\right. \\
& \left.-f_{s}^{\alpha}(\mathbf{t}, \mathbf{x})\left(1-f_{s^{\prime}}^{\beta}(\mathbf{t}, \mathbf{x})\right) \int_{C_{\beta}} S_{s, s^{\prime}}\left(\mathbf{k}, \mathbf{k}^{\prime}\right) d \mathbf{k}^{\prime}\right] .
\end{aligned}
$$

So, defining

$$
A_{s, s^{\prime}}^{\alpha, \beta}=\int_{C_{\alpha}}\left[\int_{C_{\beta}} S_{s, s^{\prime}}\left(\mathbf{k}, \mathbf{k}^{\prime}\right) d \mathbf{k}^{\prime}\right] d \mathbf{k},
$$

it is immediate to verify that

$$
\int_{C_{\alpha}} Q\left(f_{s}, f_{-s}\right) d \mathbf{k} \approx \sum_{s^{\prime}} \sum_{\beta=1}^{N}\left[A_{s^{\prime}, s}^{\beta, \alpha}\left(1-f_{s}^{\alpha}(\mathbf{t}, \mathbf{x})\right) f_{s^{\prime}}^{\beta}(\mathbf{t}, \mathbf{x})-A_{s, s^{\prime}}^{\alpha, \beta} f_{s}^{\alpha}(\mathbf{t}, \mathbf{x})\left(1-f_{s^{\prime}}^{\beta}(\mathbf{t}, \mathbf{x})\right)\right] .
$$

In order to complete the numerical treatment of the Boltzmann equation it is necessary to specify the numerical domain in the $\mathbf{k}$-space. We choose the circle $|\mathbf{k}| \leq k_{\max }$, 


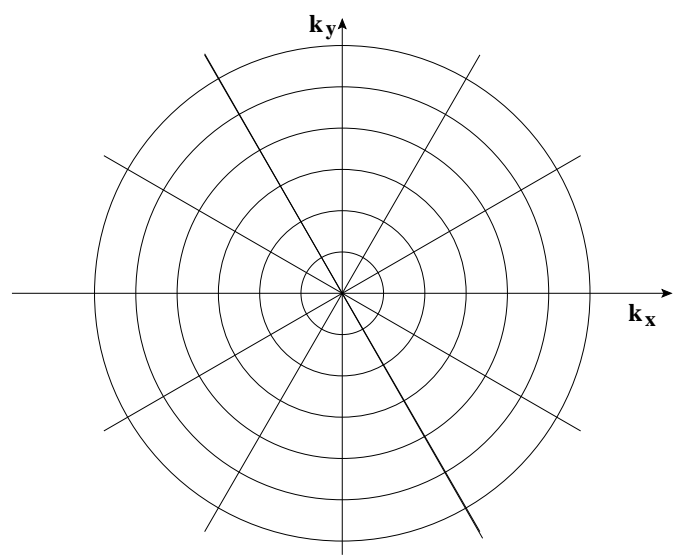

Figure 1: Grid in polar coordinates used for the discretization of the k-domain.

where $k_{\max }$ is a fixed maximum value such that $f_{+}$and $1-f_{-}$are negligible for all $\mathbf{k}$ such that $|\mathbf{k}|>k_{\max }$. The parameter $k_{\max }$ is checked a posteriori by means of numerical experiments.

We use modified polar coordinates by setting $\mathbf{k}=\sqrt{p}(\cos \vartheta, \sin \vartheta)$, and we introduce a regular decomposition (see Fig. 1). Hence the cell $C_{\alpha}$ is defined by the inequalities

$$
0 \leq p_{\alpha}<p<p_{\alpha}+\Delta p \leq \sqrt{k_{\max }} \text { and } 0 \leq \vartheta_{\alpha}<\vartheta<\vartheta_{\alpha}+\Delta \vartheta \leq 2 \pi
$$

where $\Delta p$ and $\Delta \vartheta$ are constant for every cell $C_{\alpha}$.

The use of the variable $\sqrt{p}$ instead of the modulus of $\mathbf{k}$ is aimed to having integrable parameters arising from the force term of the Boltzmann equation. Now, one can obtain the explicit expression of the numerical parameters $A_{s, s^{\prime}}^{\alpha, \beta}$ which are given in the Appendix $B$. The integrals involving the electric field are derived by means of the same technique used in [9].

We observe that considering the distribution $f_{h}$ of holes in the VB instead of the one of electrons, one gets that also $f_{h} \approx 0$ for all $\mathbf{k} \notin \Omega$, similarly to $f_{+}$, and this helps the numerical approach that we use for the integration of the transport equations.

From the approximation of the distribution functions, the average values of density, velocity and energy are reconstructed as follows

$$
\begin{aligned}
\rho_{e}(\mathbf{t}, \mathbf{x}):=\frac{2}{(2 \pi)^{2}} \int f_{+}(\mathbf{t}, \mathbf{x}, \mathbf{k}) d \mathbf{k} \approx \frac{2}{(2 \pi)^{2}} \sum_{\alpha=1}^{N} \operatorname{meas}\left(C_{\alpha}\right) f_{+}^{\alpha}(\mathbf{t}, \mathbf{x}), \\
\left\langle\mathbf{v}_{e}>(\mathbf{t}, \mathbf{x}):=\frac{1}{\rho_{e}(\mathrm{t}, \mathbf{x})} \frac{2}{(2 \pi)^{2}} \int f_{+}(\mathbf{t}, \mathbf{x}, \mathbf{k}) \mathbf{v}_{+}(\mathbf{k}) d \mathbf{k}\right. \\
\approx \frac{1}{\rho_{e}(\mathrm{t}, \mathbf{x})} \frac{2}{(2 \pi)^{2}} \sum_{\alpha=1}^{N}\left[\int_{C_{\alpha}} \mathbf{v}_{+}(\mathbf{k}) d \mathbf{k}\right] f_{+}^{\alpha}(\mathbf{t}, \mathbf{x}),
\end{aligned}
$$




$$
\begin{aligned}
& <\varepsilon_{e}>(\mathrm{t}, \mathbf{x}):=\frac{1}{\rho_{e}(\mathrm{t}, \mathbf{x})} \frac{2}{(2 \pi)^{2}} \int f_{+}(\mathbf{t}, \mathbf{x}, \mathbf{k}) \varepsilon_{+}(\mathbf{k}) d \mathbf{k} \\
& \approx \frac{1}{\rho_{e}(\mathbf{t}, \mathbf{x})} \frac{2}{(2 \pi)^{2}} \sum_{\alpha=1}^{N}\left[\int_{C_{\alpha}} \varepsilon_{+}(\mathbf{k}) d \mathbf{k}\right] f_{+}^{\alpha}(\mathbf{t}, \mathbf{x}), \\
& \rho_{h}(\mathbf{t}, \mathbf{x}):=\frac{2}{(2 \pi)^{2}} \int\left(1-f_{-}(\mathbf{t}, \mathbf{x},-\mathbf{k})\right) d \mathbf{k} \approx \frac{2}{(2 \pi)^{2}} \sum_{\alpha=1}^{N} \operatorname{meas}\left(C_{\alpha}\right)\left(1-f_{-}^{\alpha}(\mathbf{t}, \mathbf{x})\right), \\
& <\mathbf{v}_{h}>(\mathrm{t}, \mathbf{x}):=\frac{1}{\rho_{h}(\mathrm{t}, \mathbf{x})} \frac{2}{(2 \pi)^{2}} \int f_{h}(\mathrm{t}, \mathbf{x}, \mathbf{k}) \mathbf{v}_{h}(\mathbf{k}) d \mathbf{k} \\
& =\frac{1}{\rho_{h}(\mathbf{t}, \mathbf{x})} \frac{2}{(2 \pi)^{2}} \int\left(1-f_{-}(\mathbf{t}, \mathbf{x},-\mathbf{k})\right) \mathbf{v}_{h}(\mathbf{k}) d \mathbf{k} \\
& =\frac{1}{\rho_{h}(\mathbf{t}, \mathbf{x})} \frac{2}{(2 \pi)^{2}} \int\left(1-f_{-}(\mathbf{t}, \mathbf{x},-\mathbf{k})\right) \mathbf{v}_{+}(\mathbf{k}) d \mathbf{k} \\
& \approx-\frac{1}{\rho_{h}(\mathbf{t}, \mathbf{x})} \frac{2}{(2 \pi)^{2}} \sum_{\alpha=1}^{N}\left[\int_{C_{\alpha}} \mathbf{v}_{+}(\mathbf{k}) d \mathbf{k}\right]\left(1-f_{-}^{\alpha}(\mathbf{t}, \mathbf{x})\right), \\
& <\varepsilon_{h}>(\mathbf{t}, \mathbf{x}):=\frac{1}{\rho_{h}(\mathbf{t}, \mathbf{x})} \frac{2}{(2 \pi)^{2}} \int\left(1-f_{-}(\mathbf{t}, \mathbf{x},-\mathbf{k})\right) \varepsilon_{+}(\mathbf{k}) d \mathbf{k} \\
& \approx \frac{1}{\rho_{h}(\mathrm{t}, \mathbf{x})} \frac{2}{(2 \pi)^{2}} \sum_{\alpha=1}^{N}\left[\int_{C_{\alpha}} \varepsilon_{+}(\mathbf{k}) d \mathbf{k}\right]\left(1-f_{-}^{\alpha}(\mathbf{t}, \mathbf{x})\right) .
\end{aligned}
$$

The above integrals are easily evaluated by taking into account that

$$
\begin{aligned}
& \operatorname{meas}\left(C_{\alpha}\right)=\frac{1}{2} \Delta p \Delta \vartheta, \\
& \int_{C_{\alpha}} \mathbf{v}_{s} d \mathbf{k}=s v_{F} \Delta p\left(\cos \left(\vartheta+\frac{\Delta \vartheta}{2}\right) \sin \frac{\Delta \vartheta}{2}, \sin \left(\vartheta+\frac{\Delta \vartheta}{2}\right) \sin \frac{\Delta \vartheta}{2}\right), \\
& \int_{C_{\alpha}} \varepsilon_{S}(\mathbf{k}) d \mathbf{k}=\frac{1}{3} s v_{F} \hbar \Delta \vartheta\left[\sqrt{\left(p_{\alpha}+\Delta p\right)^{3}}-\sqrt{\left(p_{\alpha}\right)^{3}}\right] .
\end{aligned}
$$

Regarding the current density $\mathbf{J}$, it is given by the sum of the contribution from electrons in the CB band and holes in the VB

$$
\mathbf{J}=\mathbf{J}_{e}+\mathbf{J}_{h} .
$$

The term $\mathbf{J}_{e}$ is given by $-e \rho_{e}<\mathbf{v}_{e}>$ while

$$
\mathbf{J}_{h}=e \frac{2}{(2 \pi)^{2}} \int\left(1-f_{-}(\mathbf{t}, \mathbf{x},-\mathbf{k})\right) \mathbf{v}_{h}(\mathbf{k}) d \mathbf{k}=-e \frac{2}{(2 \pi)^{2}} \int\left(1-f_{-}(\mathbf{t}, \mathbf{x}, \mathbf{k})\right) \mathbf{v}_{h}(\mathbf{k}) d \mathbf{k} .
$$

Remark 3.1. The fact that the valence band is populated with a huge number of electrons (recall that $f_{-} \approx 1$ when $|\mathbf{k}| \gg 1$ ), requires in a DSMC the introduction of a prohibitive 
number of particles. To overcome the problem one can consider holes in the valence band. However, such a choice has the drawback to make ambiguous the interband scattering mechanism, as explained below.

Let us consider an electron having energy $\varepsilon$. If it happens that $0<\varepsilon<\hbar \omega^{(v)}$ and an emission of a $v$ th phonon occurs, where $v$ is OP or $\mathrm{K}$, then an interband scattering event takes place. After the transition the electron will have a state determined according to the $v$ th transition rate (see [8]) and the new energy.

If we try to rewrite this interband scattering in terms of holes, instead of electrons, in the valence band, then the electron recombines with a hole. In order to have a recombination we need to find a hole having exactly the energy $\hbar \omega^{(v)}-\varepsilon$ and this can be achieved only in an approximate, often roughly, way.

The treatment of the case when a hole has an energy $0<\varepsilon<\hbar \omega^{(v)}$ is even worse. After the scattering event the considered hole can disappear through two different mechanisms: an electron coming from the conduction band recombines with a hole or an electron of the valence band occupies the position of the hole leaving behind an other hole. The only viable approach to discriminate between the two occurrences is to describe both electrons and holes in the valence band and again we have to face the question related to the huge number of required simulation particles.

A similar ambiguity arises for the description of electron-hole pair creation.

The above considerations clearly indicate that the deterministic methods, as the DG adopted in the present paper, are the only reasonable ones for solving the transport equations for charge carriers in graphene in the presence of interband scatterings.

\section{Numerical results}

The physical situation we are going to simulate is that of a strip of graphene which is infinitely long in the transversal direction with respect to that of the electric field (see Fig. 2). This allows us to look for solutions which are not depending on space and to avoid any effect related to the boundary conditions.

We have considered several values of the Fermi energy and different applied electric fields.

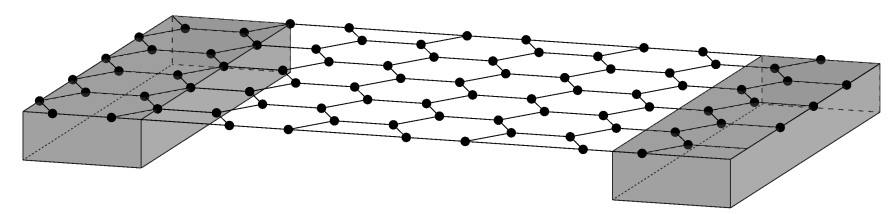

Figure 2: Schematic representation of a suspended monolayer graphene. In the direction parallel to the contacts the material is infinitely long. In each contact there is a constant electrostatic potential. 


\subsection{Case $\varepsilon_{F}=0 \mathrm{eV}$}

This case represents a pristine graphene and it is the most challenging for the correct evaluation of the role of the interband scattering. In Fig. 3 there is shown the density versus time (in logarithmic scale). During a long transient of a few hundreds of picoseconds, the concentration of both charge carriers increases up to saturation values depending on the applied electric field. The effect is that of carrier multiplications and it is due to the fact that the generation term overcomes for a long time that of recombination with the results of the creation of electron-hole pairs. Only after about 200 picosecond the conduction band is populated enough so that Pauli's exclusion principle becomes so efficient to prevent the formation of additional electron-hole pairs. A similar phenomenon had have been observed for example in [28] and in [29] analyzing the optical properties of graphene in view of possible applications to solar cells.

We remark that when $\varepsilon_{F}=0 \mathrm{eV}$, the electron and hole densities are the same. For this reason only the electron density is plotted. Of course, neglecting the interband scatterings, the densities do not change with time. For comparison, also the densities without interband scattering are reported in the figures.
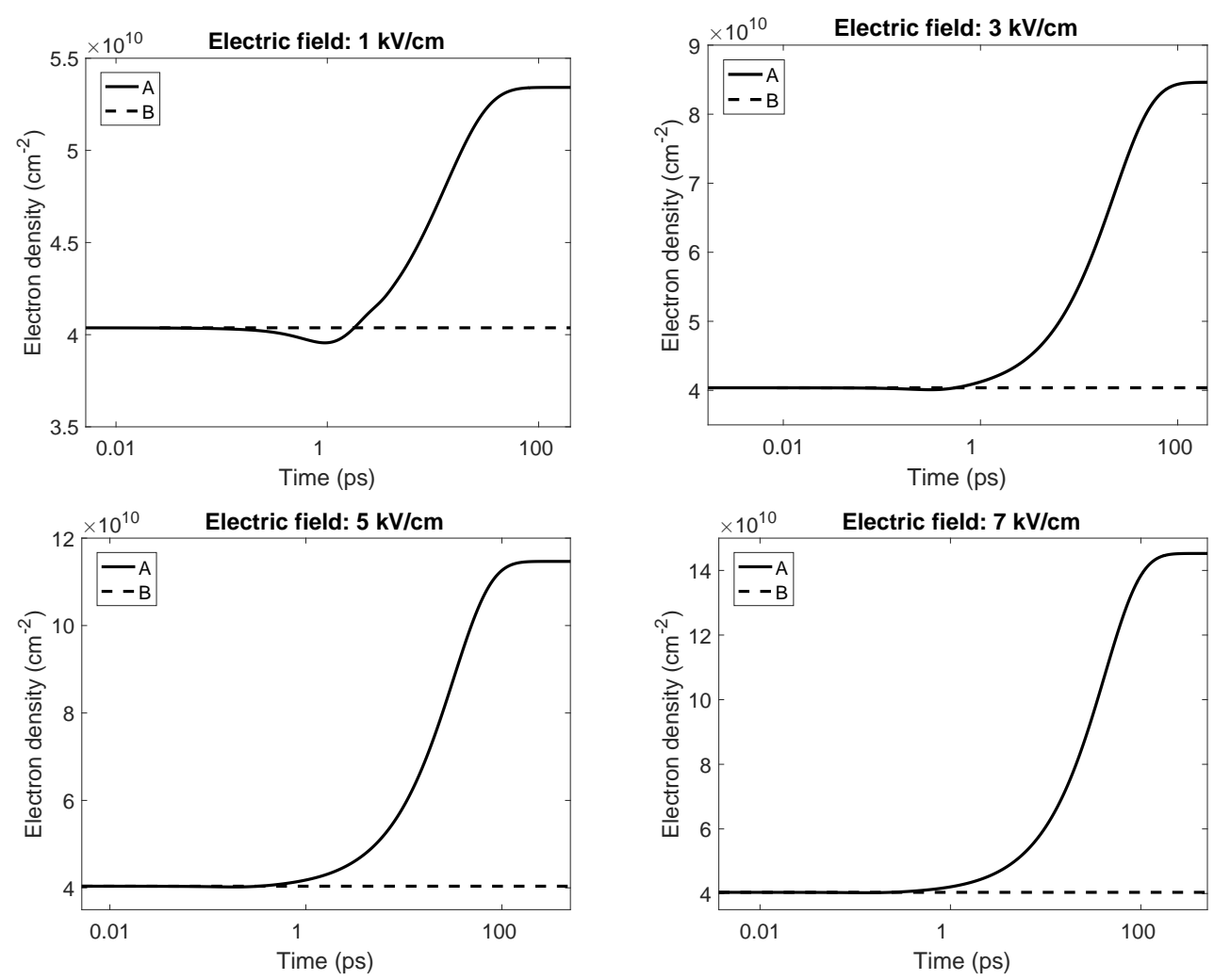

Figure 3: Electron density versus time when $\varepsilon_{F}=0 \mathrm{eV}$ under an applied electric field of $1,3,5,7 \mathrm{kV} / \mathrm{cm}$ with (A) and without (B) interband scatterings. 

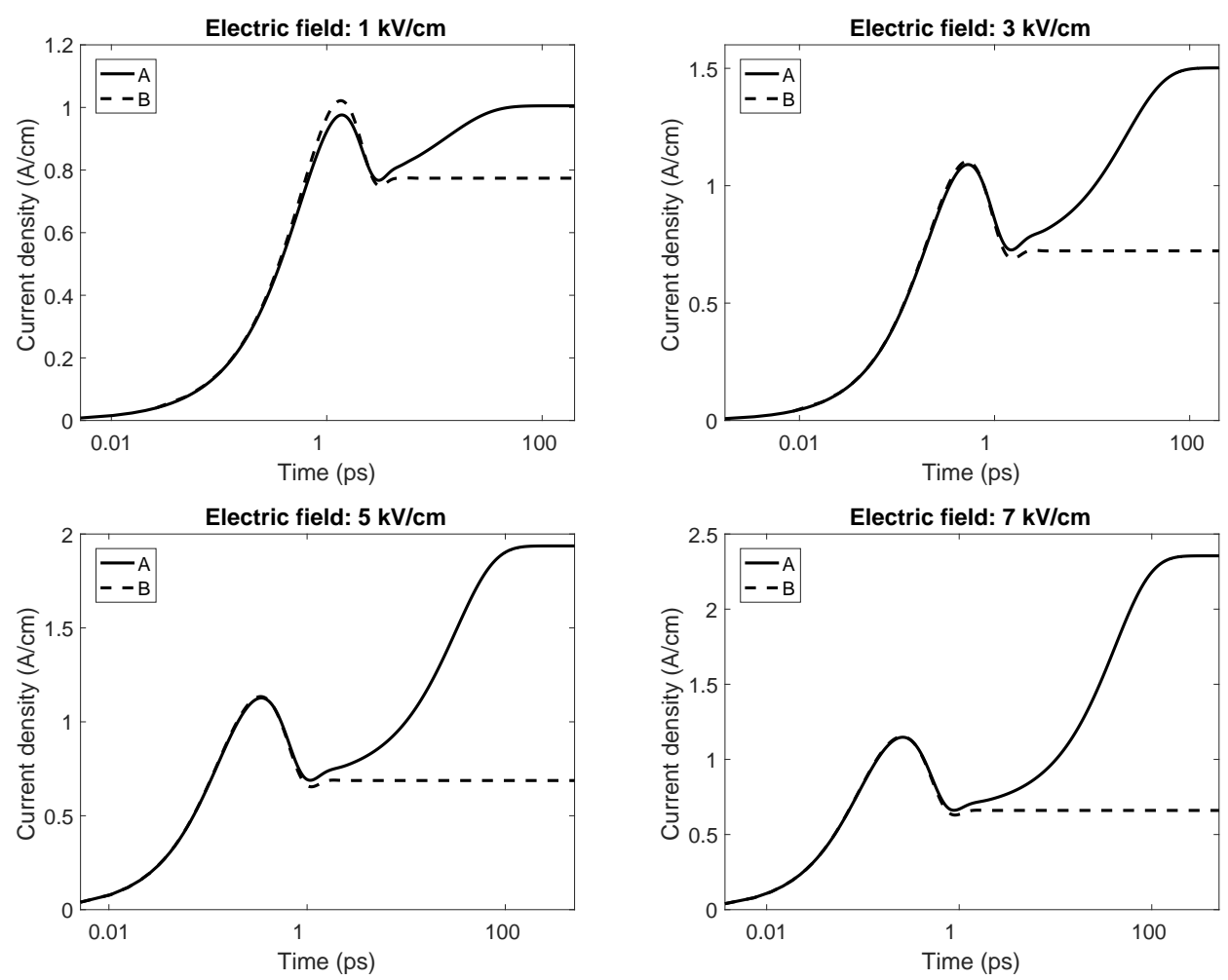

Figure 4: Total current versus time when $\varepsilon_{F}=0 \mathrm{eV}$ under an applied electric field of $1,3,5,7 \mathrm{kV} / \mathrm{cm}$ with (A) and without $(B)$ interband scatterings.

In Fig. 4 the total current (electrons plus holes) is plotted versus time. Similarly to the density, the current increases with time, apart an initial transient. The carrier multiplication leads to a considerably higher current with respect to the case when only intraband scatterings are included. The steady total current versus the electric field is reported in Fig. 5. Note that neglecting the interband scatterings introduces an error of about $400 \%$ for the highest electric field considered in the simulation $(10 \mathrm{kV} / \mathrm{cm})$.

\subsection{Case $\varepsilon_{F}=0.1 \mathrm{eV}$}

In order to assess the influence of the Fermi level, the case with $\varepsilon_{F}=0.1 \mathrm{eV}$ has been also analyzed. The results are shown in Figs. 6, 7, 8. First we note that the electron and hole densities are no longer equal. Moreover, as expected, the difference is about two orders of magnitude because electrons are the majority carriers when the Fermi energy is positive. The maximum change obtained in the simulations with respect to the case without interband scatterings is less than $1 \%$ for electrons and more than $90 \%$ for holes. This means that neglecting the interband effects leads to severely underestimate the hole density. Regarding the discrepancy in the current, for the electric field of $10 \mathrm{kV} / \mathrm{cm}$ one has a relative difference of about $22 \%$. 


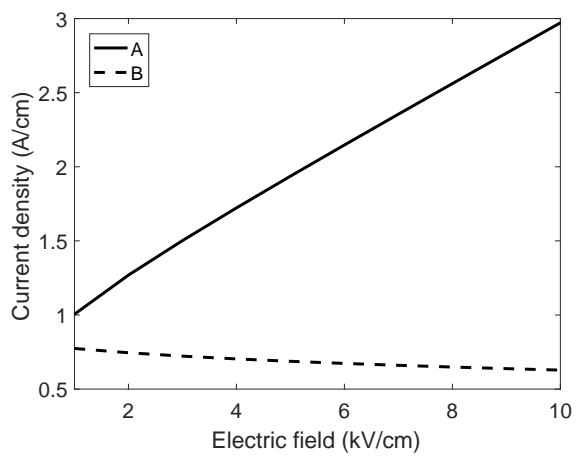

Figure 5: Steady total current versus electric field when $\varepsilon_{F}=0 \mathrm{eV}$ with $(\mathrm{A})$ and without $(\mathrm{B})$ interband scatterings.
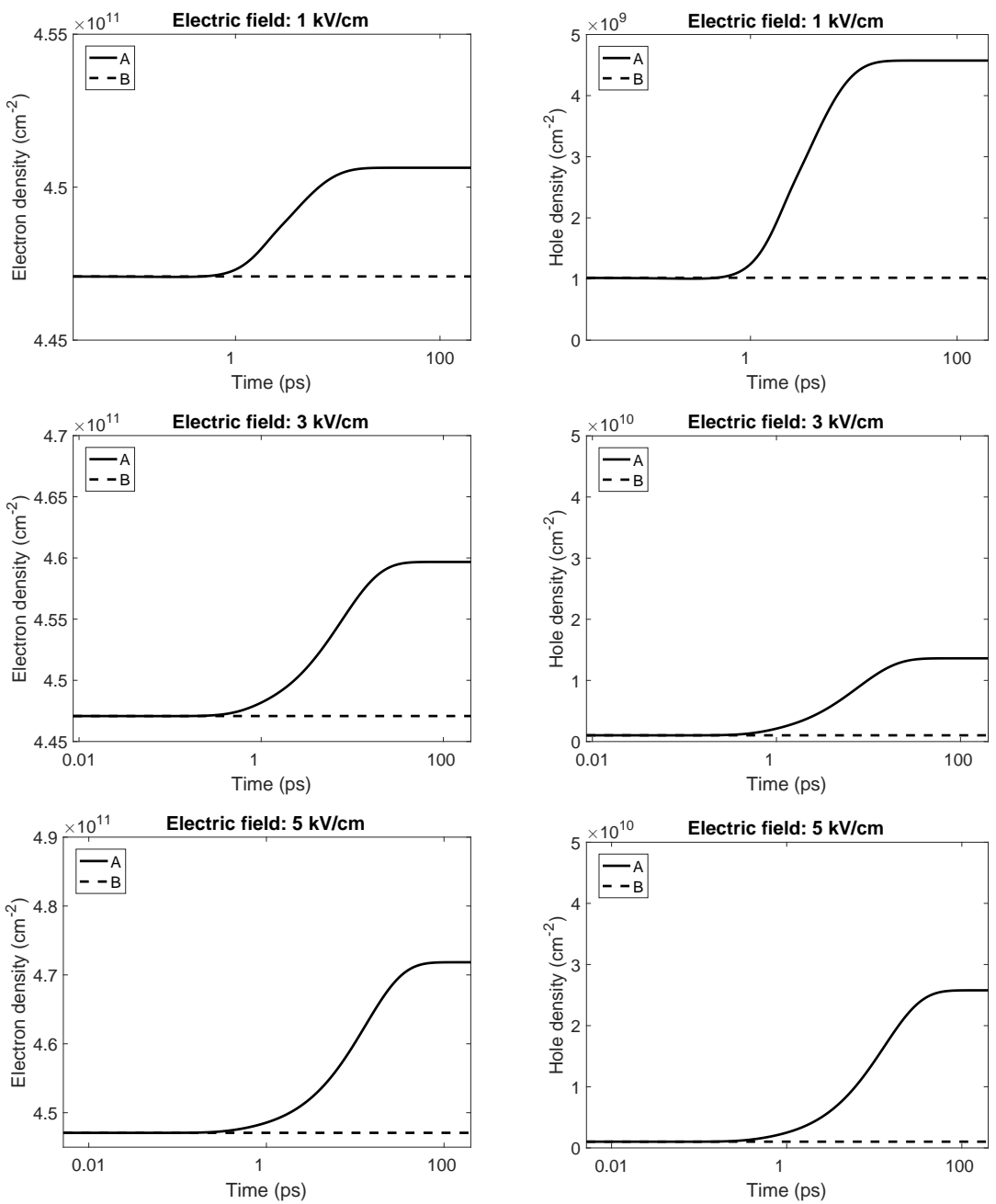

Figure 6: Electron density (left column) and hole density (right column) versus time when $\varepsilon_{F}=0.1 \mathrm{eV}$ under an applied field of $1,3,5 \mathrm{kV} / \mathrm{cm}$ with (A) and without (B) interband scatterings. 

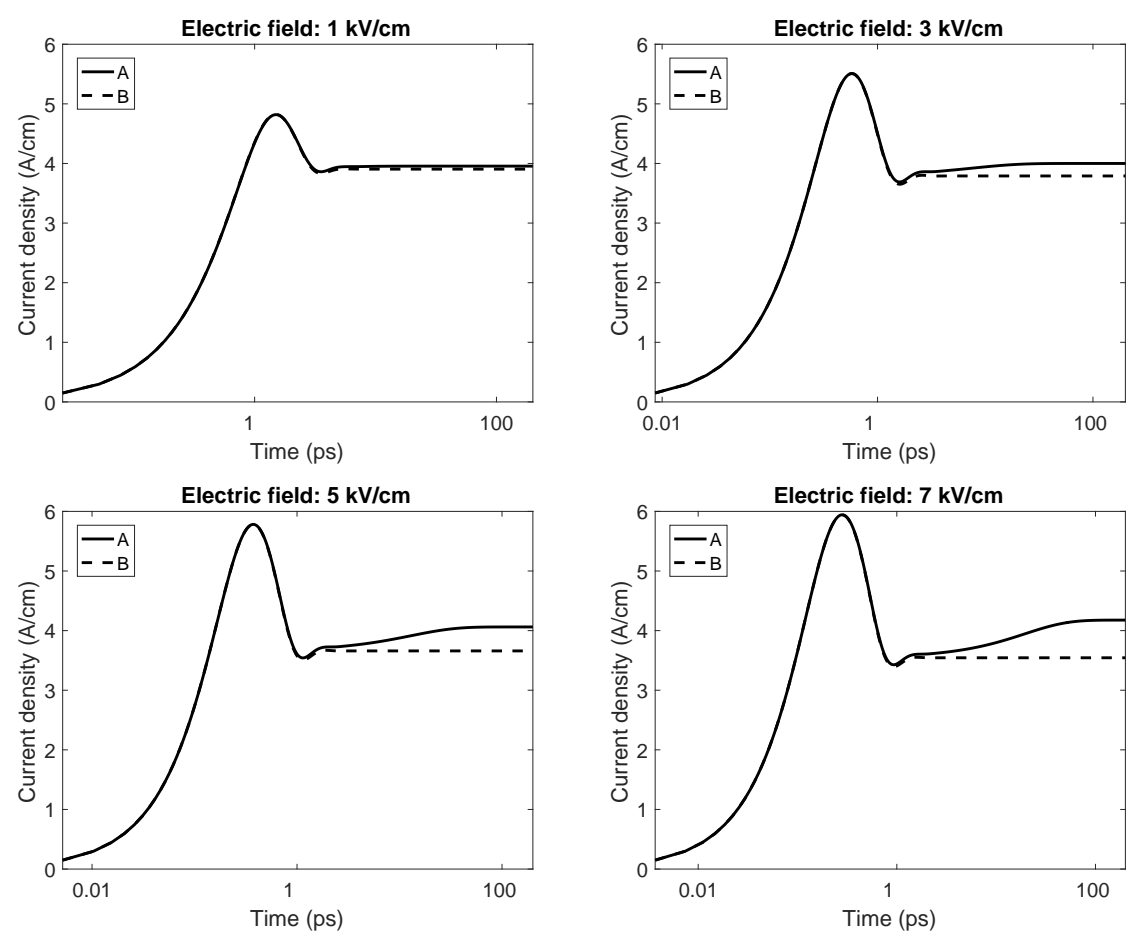

Figure 7: Total current versus time when $\varepsilon_{F}=0.1 \mathrm{eV}$ under an applied electric field of $1,3,5,7 \mathrm{kV} / \mathrm{cm}$ with (A) and without (B) interband scatterings.

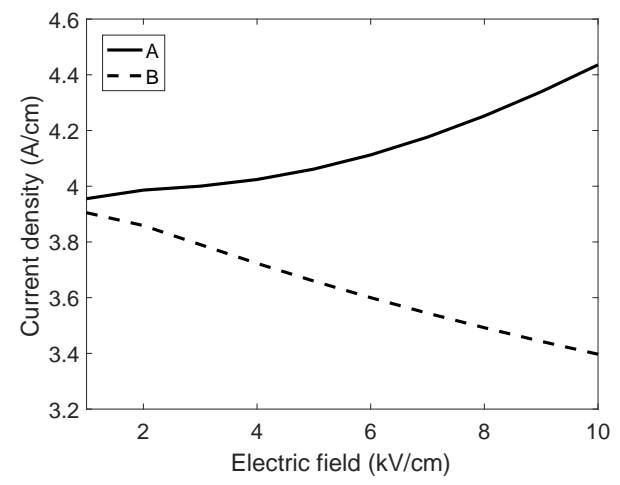

Figure 8: Steady total current versus electric field when $\varepsilon_{F}=0.1 \mathrm{eV}$.

If the reverse sign of Fermi energy is considered, we have the same results by interchanging the role of electrons and holes.

\subsection{Case $\varepsilon_{F}=0.2 \mathrm{eV}$}

Finally, the case with $\varepsilon_{F}=0.2 \mathrm{eV}$ has been analyzed. The results are shown in Figs. 9, 10, 11. The transient is shorter: the Pauli exclusion principle drastically reduces the forma- 



Figure 9: Electron density (left column) and hole density (right column) versus time when $\varepsilon_{F}=0.2 \mathrm{eV}$ under an applied electric field of $1,3,5 \mathrm{kV} / \mathrm{cm}$.

tion of electron-hole pairs already after few picoseconds. The electron density remains practically unchanged, while that of holes increases more than one order of magnitude. However, the difference between the majority and minority charges is so high that, for Fermi levels greater or equal to $0.2 \mathrm{eV}$, the simulation can be performed with a good accuracy disregarding the interband effects. If the reverse sign of Fermi energy is considered, we have again the same results by interchanging the role of electrons and holes. 

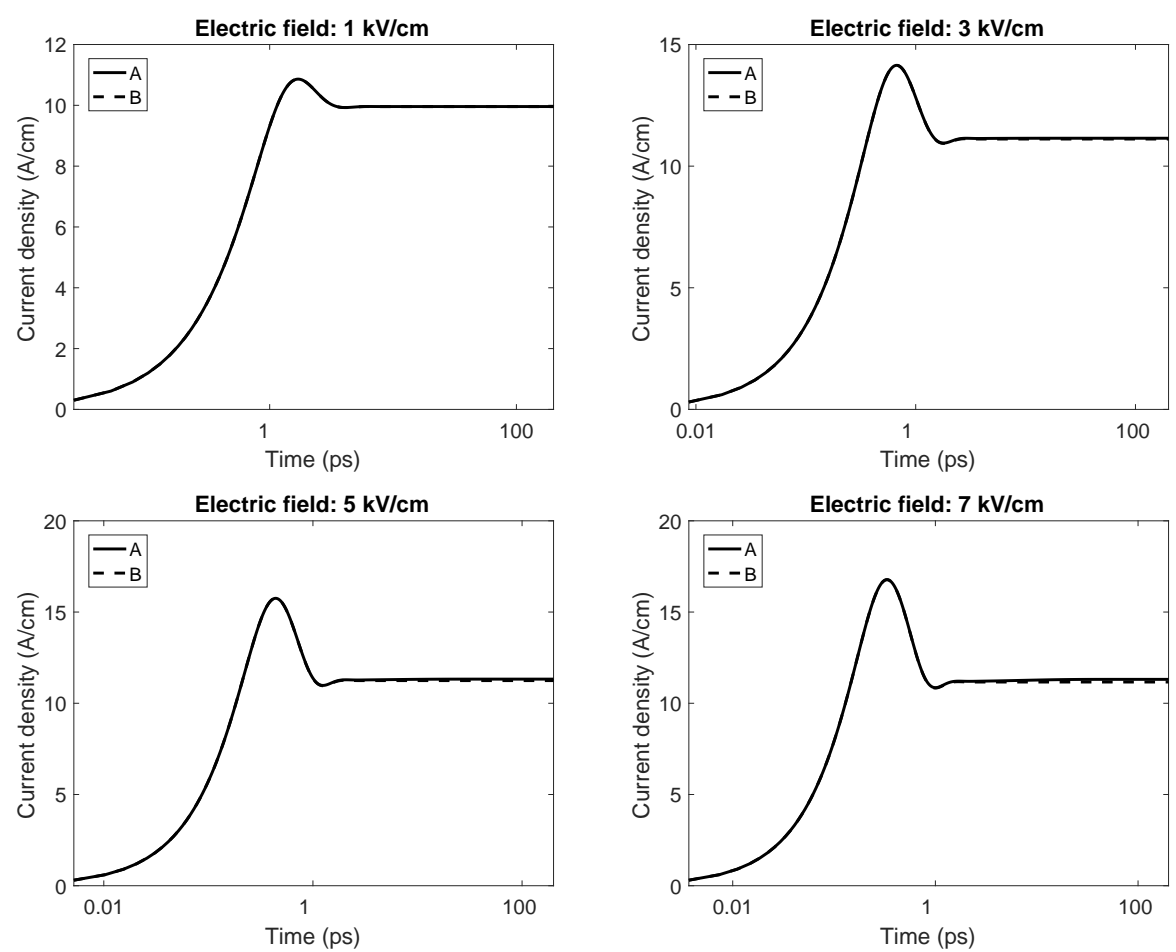

Figure 10: Total current versus time when $\varepsilon_{F}=0.2 \mathrm{eV}$ under an applied electric field of $1,3,5,7 \mathrm{kV} / \mathrm{cm}$.

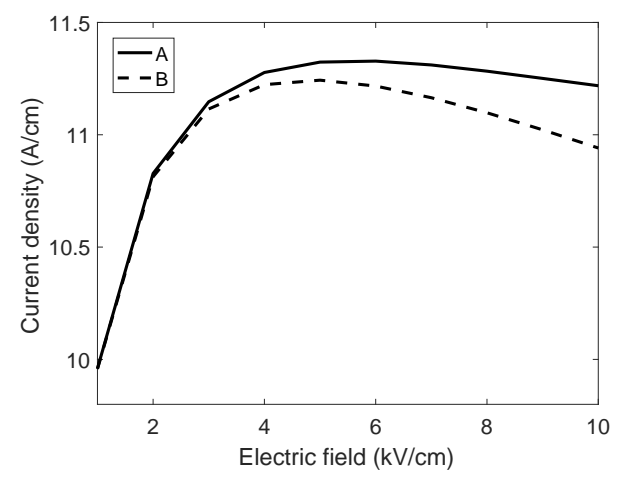

Figure 11: Steady total current versus electric field when $\varepsilon_{F}=0.2 \mathrm{eV}$.

\section{Conclusions}

Charge transport in suspended monolayer graphene has been simulated by a numerical deterministic approach, based on a discontinuous Galerkin (DG) method, for solving the semiclassical Boltzmann equations for electrons and holes. Both the conduction and valence bands have been included and the interband scatterings have been taken into account. 
The importance of the interband scatterings has been accurately evaluated for several values of the Fermi energy. It is found out that the inclusion of the interband scatterings produces, with zero Fermi energy, huge variations in the current, while, as expected, the effect of the interband scatterings becomes negligible by increasing the absolute value of the Fermi energy. When $\varepsilon_{F}$ is greater than $0.2 \mathrm{eV}$, the interband effects can be eliminated without any significant consequence in the simulations.

\section{Acknowledgments}

The authors acknowledge the financial support by progetto FIR 2016-2018 Modellistica, simulazione e ottimizzazione del trasporto di cariche in strutture a bassa dimensionalità, University of Catania, and by INDAM (GNFM).

\section{Appendix A}

\section{Deduction of the Boltzmann equation for holes}

Starting from the Boltzmann equation for $f_{-}$one has

$$
\partial_{\mathrm{t}} f_{-}(\mathbf{t}, \mathbf{x}, \mathbf{k})+\mathbf{v}_{-}(\mathbf{k}) \cdot \nabla_{\mathbf{x}} f_{-}(\mathbf{t}, \mathbf{x}, \mathbf{k})-\frac{e}{\hbar} \mathbf{E} \cdot \nabla_{\mathbf{k}} f_{-}(\mathbf{t}, \mathbf{x}, \mathbf{k})=Q\left(f_{-}, f_{+}\right)(\mathbf{t}, \mathbf{x}, \mathbf{k}),
$$

where

$$
\mathbf{v}_{-}(\mathbf{k})=\frac{1}{\hbar} \nabla_{\mathbf{k} \varepsilon_{-}}(\mathbf{k})=-\frac{1}{\hbar} \hbar v_{F} \nabla_{\mathbf{k}}|\mathbf{k}|=-v_{F} \frac{\mathbf{k}}{|\mathbf{k}|}
$$

and

$$
\begin{aligned}
Q\left(f_{-}, f_{+}\right)= & \int_{\mathbb{R}^{2}}\left[S_{-,-}\left(\mathbf{k}^{\prime}, \mathbf{k}\right) f_{-}\left(\mathbf{k}^{\prime}\right)\left(1-f_{-}(\mathbf{k})\right)-S_{-,-}\left(\mathbf{k}, \mathbf{k}^{\prime}\right) f_{-}(\mathbf{k})\left(1-f_{-}\left(\mathbf{k}^{\prime}\right)\right)\right] d \mathbf{k}^{\prime} \\
& +\int_{\mathbb{R}^{2}}\left[S_{+,-}\left(\mathbf{k}^{\prime}, \mathbf{k}\right) f_{+}\left(\mathbf{k}^{\prime}\right)\left(1-f_{-}(\mathbf{k})\right)-S_{-,+}\left(\mathbf{k}, \mathbf{k}^{\prime}\right) f_{-}(\mathbf{k})\left(1-f_{+}\left(\mathbf{k}^{\prime}\right)\right)\right] d \mathbf{k}^{\prime} .
\end{aligned}
$$

We would like to get the distribution of missing electron states, that are the holes. For these quasi-particles the following definitions hold

$$
\mathbf{k}_{h}=-\mathbf{k}_{e}, \quad \varepsilon_{h}\left(\mathbf{k}_{h}\right)=-\varepsilon_{-}\left(\mathbf{k}_{e}\right) .
$$

Thus the dispersion relation and the distribution function for holes can be written respectively as

$$
\varepsilon_{h}(\mathbf{k})=\hbar v_{F}|\mathbf{k}|, \quad f_{h}(\mathbf{t}, \mathbf{x}, \mathbf{k})=1-f_{-}(\mathbf{t}, \mathbf{x}, \mathbf{k}),
$$

and the group velocity is

$$
\mathbf{v}_{h}(\mathbf{k})=\frac{1}{\hbar} \nabla_{\mathbf{k}} \varepsilon_{h}(\mathbf{k})=v_{F} \frac{\mathbf{k}}{|\mathbf{k}|} .
$$


Relabeling the variable $\mathbf{k}$ of equation (A.1) with $\mathbf{- k}$, the Boltzmann equation for the distribution function of missing electrons is

$$
\begin{aligned}
& \partial_{\mathrm{t}}\left(1-f_{-}(\mathbf{t}, \mathbf{x},-\mathbf{k})\right)+\mathbf{v}_{-}(-\mathbf{k}) \cdot \nabla_{\mathbf{x}}\left(1-f_{-}(\mathbf{t}, \mathbf{x},-\mathbf{k})\right)-\frac{e}{\hbar} \mathbf{E} \cdot \nabla_{-\mathbf{k}}\left(1-f_{-}(\mathbf{t}, \mathbf{x},-\mathbf{k})\right) \\
= & -Q\left(f_{-}, f_{+}\right)(\mathbf{t}, \mathbf{x},-\mathbf{k}) .
\end{aligned}
$$

After observing that

$$
\mathbf{v}_{-}(-\mathbf{k})=-v_{F} \frac{-\mathbf{k}}{|-\mathbf{k}|}=\mathbf{v}_{h}(\mathbf{k})
$$

we have

$$
\partial_{\mathrm{t}} f_{h}(\mathbf{t}, \mathbf{x}, \mathbf{k})+\mathbf{v}_{h}(\mathbf{k}) \cdot \nabla_{\mathbf{x}} f_{h}(\mathbf{t}, \mathbf{x}, \mathbf{k})+\frac{e}{\hbar} \mathbf{E} \cdot \nabla_{\mathbf{k}} f_{h}(\mathbf{t}, \mathbf{x}, \mathbf{k})=-Q\left(f_{-}, f_{+}\right)(\mathbf{t}, \mathbf{x},-\mathbf{k}) .
$$

Regarding the collisional term, we have

$$
\begin{aligned}
& -Q\left(f_{-}, f_{+}\right)(\mathbf{t}, \mathbf{x},-\mathbf{k}) \\
= & -\int_{\mathbb{R}^{2}}\left[S_{-,-}\left(\mathbf{k}^{\prime},-\mathbf{k}\right) f_{-}\left(\mathbf{k}^{\prime}\right)\left(1-f_{-}(-\mathbf{k})\right)-S_{-,-}\left(-\mathbf{k}, \mathbf{k}^{\prime}\right) f_{-}(-\mathbf{k})\left(1-f_{-}\left(\mathbf{k}^{\prime}\right)\right)\right] d \mathbf{k}^{\prime} \\
& -\int_{\mathbb{R}^{2}}\left[S_{+,-}\left(\mathbf{k}^{\prime},-\mathbf{k}\right) f_{+}\left(\mathbf{k}^{\prime}\right)\left(1-f_{-}(-\mathbf{k})\right)-S_{-,+}\left(-\mathbf{k}, \mathbf{k}^{\prime}\right) f_{-}(-\mathbf{k})\left(1-f_{+}\left(\mathbf{k}^{\prime}\right)\right)\right] d \mathbf{k}^{\prime} \\
= & -\int_{\mathbb{R}^{2}}\left[S_{-,-}\left(\mathbf{k}^{\prime},-\mathbf{k}\right)\left(1-f_{h}\left(-\mathbf{k}^{\prime}\right)\right) f_{h}(\mathbf{k})-S_{-,-}\left(-\mathbf{k}, \mathbf{k}^{\prime}\right)\left(1-f_{h}(\mathbf{k})\right) f_{h}\left(-\mathbf{k}^{\prime}\right)\right] d \mathbf{k}^{\prime} \\
& -\int_{\mathbb{R}^{2}}\left[S_{+,-}\left(\mathbf{k}^{\prime},-\mathbf{k}\right) f_{+}\left(\mathbf{k}^{\prime}\right) f_{h}(\mathbf{k})-S_{-,+}\left(-\mathbf{k}, \mathbf{k}^{\prime}\right)\left(1-f_{h}(\mathbf{k})\right)\left(1-f_{+}\left(\mathbf{k}^{\prime}\right)\right)\right] d \mathbf{k}^{\prime} \\
= & -\int_{\mathbb{R}^{2}}\left[S_{-,-}\left(-\mathbf{k}^{\prime},-\mathbf{k}\right) f_{h}(\mathbf{k})\left(1-f_{h}\left(\mathbf{k}^{\prime}\right)\right)-S_{-,-}\left(-\mathbf{k},-\mathbf{k}^{\prime}\right) f_{h}\left(\mathbf{k}^{\prime}\right)\left(1-f_{h}(\mathbf{k})\right)\right] d \mathbf{k}^{\prime} \\
& -\int_{\mathbb{R}^{2}}\left[S_{+,-}\left(\mathbf{k}^{\prime},-\mathbf{k}\right) f_{+}\left(\mathbf{k}^{\prime}\right) f_{h}(\mathbf{k})-S_{-,+}\left(-\mathbf{k}, \mathbf{k}^{\prime}\right)\left(1-f_{h}(\mathbf{k})\right)\left(1-f_{+}\left(\mathbf{k}^{\prime}\right)\right)\right] d \mathbf{k}^{\prime},
\end{aligned}
$$

where the last step is obtained by performing a change of variables $\mathbf{k}^{\prime} \rightarrow-\mathbf{k}^{\prime}$. Definitely the collisional term writes

$$
-Q\left(f_{-}, f_{+}\right)(\mathbf{t}, \mathbf{x},-\mathbf{k})=Q_{h h}\left(f_{h}, f_{h}\right)(t, \mathbf{x}, \mathbf{k})+Q_{h e}\left(f_{h}, f_{+}\right)(\mathbf{t}, \mathbf{x}, \mathbf{k}),
$$

where, omitting the dependencies on $t$ and $\mathbf{x}$,

$$
\begin{aligned}
Q_{h h} & =-\int_{\mathbb{R}^{2}}\left[S_{-,-}\left(-\mathbf{k}^{\prime},-\mathbf{k}\right) f_{h}(\mathbf{k})\left(1-f_{h}\left(\mathbf{k}^{\prime}\right)\right)-S_{-,-}\left(-\mathbf{k},-\mathbf{k}^{\prime}\right) f_{h}\left(\mathbf{k}^{\prime}\right)\left(1-f_{h}(\mathbf{k})\right)\right] d \mathbf{k}^{\prime}, \\
Q_{h e} & =-\int_{\mathbb{R}^{2}}\left[S_{+,-}\left(\mathbf{k}^{\prime},-\mathbf{k}\right) f_{+}\left(\mathbf{k}^{\prime}\right) f_{h}(\mathbf{k})-S_{-,+}\left(-\mathbf{k}, \mathbf{k}^{\prime}\right)\left(1-f_{h}(\mathbf{k})\right)\left(1-f_{+}\left(\mathbf{k}^{\prime}\right)\right)\right] d \mathbf{k}^{\prime} .
\end{aligned}
$$

Let us consider the particular case where only intraband transitions are taken into account. The equation writes

$$
\partial_{t} f_{h}(\mathbf{t}, \mathbf{x}, \mathbf{k})+\mathbf{v}_{h}(\mathbf{k}) \cdot \nabla_{\mathbf{x}} f_{h}(\mathbf{t}, \mathbf{x}, \mathbf{k})+\frac{e}{\hbar} \mathbf{E} \cdot \nabla_{\mathbf{k}} f_{h}(\mathbf{t}, \mathbf{x}, \mathbf{k})=Q_{h h}(\mathbf{t}, \mathbf{x}, \mathbf{k}) .
$$

It is possible to prove that $S_{-,-}\left(-\mathbf{k}^{\prime},-\mathbf{k}\right)=S_{-,-}\left(\mathbf{k}^{\prime}, \mathbf{k}\right)$ by using geometrical considerations on the involved angles and, therefore, the relation $S_{-,-}\left(\mathbf{k}^{\prime}, \mathbf{k}\right)=S_{+,+}\left(\mathbf{k}, \mathbf{k}^{\prime}\right)$ holds. 


\section{Appendix B}

Since the transition matrix elements $\left|G_{s, s^{\prime}}^{(v)}\left(\mathbf{k}, \mathbf{k}^{\prime}\right)\right|$ depend only on the convex angle between $\mathbf{k}$ and $\mathbf{k}^{\prime}$, we can write

$$
\begin{aligned}
& A_{s, s^{\prime}}^{\alpha, \beta}=\frac{1}{4} \sum_{v} \Gamma_{s, s^{\prime}}^{(v)}(\alpha, \beta) \int_{p_{\alpha}}^{p_{\alpha}+\Delta p} \int_{p_{\beta}}^{p_{\beta}+\Delta p}\left[\left(n_{\mathbf{q}}^{(v)}+1\right) \delta\left(s^{\prime} \hbar v_{F} \sqrt{p^{\prime}}-s \hbar v_{F} \sqrt{p}+\hbar \omega_{\mathbf{q}}^{(v)}\right)\right. \\
&\left.+n_{\mathbf{q}}^{(v)} \delta\left(s^{\prime} \hbar v_{F} \sqrt{p^{\prime}}-s \hbar v_{F} \sqrt{p}-\hbar \omega_{\mathbf{q}}^{(v)}\right)\right] d p^{\prime} d p
\end{aligned}
$$

where

$$
\Gamma_{s, s^{\prime}}^{(v)}(\alpha, \beta)=\int_{\vartheta_{\alpha}}^{\vartheta_{\alpha}+\Delta \vartheta} \int_{\vartheta_{\beta}}^{\vartheta_{\beta}+\Delta \vartheta}\left|G_{s, s^{\prime}}^{(v)}\left(\mathbf{k}, \mathbf{k}^{\prime}\right)\right|^{2} d \vartheta^{\prime} d \vartheta
$$

The integrals in Eq. (B.4) are elementary. The integrals in Eq. (B.3) can be solved analytically, taking into account that

$$
\begin{aligned}
I\left(\alpha, \beta, s, s^{\prime}, \pm \omega_{\mathbf{q}}^{(v)}\right) & =\frac{1}{4} \int_{p_{\alpha}}^{p_{\alpha}+\Delta p} \int_{p_{\beta}}^{p_{\beta}+\Delta p} \delta\left(s^{\prime} \hbar v_{F} \sqrt{p^{\prime}}-s \hbar v_{F} \sqrt{p} \pm \hbar \omega_{\mathbf{q}}^{(v)}\right) d p^{\prime} d p \\
& =\frac{1}{\left|s^{\prime}\right| \hbar v_{F}} \int_{\sqrt{p_{\alpha}}}^{\sqrt{p_{\alpha}+\Delta p}} \int_{\sqrt{p_{\beta}}}^{\sqrt{p_{\beta}+\Delta p}} \delta\left(r^{\prime}-\frac{s}{s^{\prime}} r \pm \frac{\omega_{\mathbf{q}}^{(v)}}{s^{\prime} v_{F}}\right) r r^{\prime} d r^{\prime} d r \\
& =\frac{1}{\hbar v_{F}} \int_{\sqrt{p_{\alpha}}}^{\sqrt{p_{\alpha}+\Delta p}}\left[\int_{\mathbb{R}} \chi_{\left[\sqrt{p_{\beta}}, \sqrt{p_{\beta}+\Delta p}\right]}\left(r^{\prime}\right) \delta\left(r^{\prime}-\frac{s}{s^{\prime}} r \pm \frac{\omega_{\mathbf{q}}^{(v)}}{s^{\prime} v_{F}}\right) r^{\prime} d r^{\prime}\right] r d r \\
& =\frac{1}{\hbar v_{F}} \int_{\sqrt{p_{\alpha}}}^{\sqrt{p_{\alpha}+\Delta p}}\left(\frac{s}{s^{\prime}} r \mp \frac{\omega_{\mathbf{q}}^{(v)}}{s^{\prime} v_{F}}\right) \chi_{\left[\sqrt{p_{\beta}}, \sqrt{p_{\beta}+\Delta p}\right]}\left(\frac{s}{s^{\prime}} r \mp \frac{\omega_{\mathbf{q}}^{(v)}}{s^{\prime} v_{F}}\right) r d r,
\end{aligned}
$$

where $\chi_{\left[\sqrt{p_{\beta}}, \sqrt{p_{\beta}+\Delta p}\right]}$ is the characteristic function in the interval $\left[\sqrt{p_{\beta}}, \sqrt{p_{\beta}+\Delta p}\right.$. Now, we define the set

$$
\left\{r \in \mathbb{R} \text { such that } \sqrt{p_{\alpha}} \leq r \leq \sqrt{p_{\alpha}+\Delta p} \text { and } \sqrt{p_{\beta}} \leq \frac{s}{s^{\prime}} r \mp \frac{\omega_{\mathbf{q}}^{(v)}}{s^{\prime} v_{F}} \leq \sqrt{p_{\beta}+\Delta p}\right\}
$$

which is the empty set or an interval $[a, b]$. In the first case the integral vanishes, and in the last case, we have

$$
I\left(\alpha, \beta, s, s^{\prime}, \pm \omega_{\mathbf{q}}^{(v)}\right)=\frac{1}{\hbar v_{F}} \int_{a}^{b}\left(\frac{s}{s^{\prime}} r^{2} \mp \frac{\omega_{\mathbf{q}}^{(v)}}{s^{\prime} v_{F}} r\right) d r,
$$

that is an elementary integral. 


\section{References}

[1] L. Barletti, Hydrodynamic equations for electrons in graphene obtained from the maximum entropy principle, J. Math. Phys., 55(8), 083303 (2014)

[2] A. H. Castro Neto, F. Guinea, N. M. R. Peres, K. S. Novoselov, A. K. Geim, The electronic properties of graphene, Rev. Mod. Phys., vol. 81, 109-162 (2009)

[3] P. Lichtenberger, O. Morandi, F. Schürrer, High-field transport and optical phonon scattering in graphene, Phys. Rev. B, 84, 045406 (2011)

[4] O. Morandi, L. Barletti, Particle Dynamics in Graphene: Collimated Beam Limit, J. of Computational and Theoretical Transport, 43(1-7), 418-432 (2014)

[5] H. Zhao, Q. Guo, F. Xia, H. Wang, Two-dimensional materials for nanophotonics application, Nanophotonics, 4, 128-142 (2015)

[6] L. Barletti, Hydrodynamic equations for an electron gas in graphene, J. Math. Industry, 6:7, https://doi.org/10.1186/s13362-016-0023-7 (2016)

[7] O. Morandi, F. Schürrer, Wigner model for quantum transport in graphene, J. Phys. A: Math. Theor., 44, 265301 (2011)

[8] V. Romano, A. Majorana, M. Coco, DSMC method consistent with the Pauli exclusion principle and comparison with deterministic solutions for charge transport in graphene, J. Comput. Phys., 302, 267-284 (2015)

[9] M. Coco, A. Majorana, V. Romano, Cross validation of discontinuous Galerkin method and Monte Carlo simulations of charge transport in graphene on substrate, Ricerche mat., 66, 201-220 (2017)

[10] A. Majorana, V. Romano, Numerical Solutions of the Spatially Homogeneous Boltzmann Equation for Electrons in n-Doped Graphene on a Substrate, J. of Computational and Theoretical Transport, 46(3), 176-185 (2017)

[11] M. Coco, G. Mascali, V. Romano, Monte Carlo Analysis of Thermal Effects in Monolayer Graphene, J. of Computational and Theoretical Transport, 45(7), 540-553 (2016)

[12] A. Majorana, G. Mascali, V. Romano, Charge transport and mobility in monolayer graphene, J. Math. Industry, 7:4, https://doi.org/10.1186/s13362-016-0027-3 (2016)

[13] Y. Cheng, I. M. Gamba, A. Majorana, C.-W. Shu, A discontinuous Galerkin solver for Boltzmann-Poisson systems in nano devices, Comput. Methods Appl. Mech. Engrg., 198(3740), 3130-3150 (2009)

[14] Y. Cheng, I. M. Gamba, A. Majorana, C.-W. Shu, A brief survey of the discontinuous Galerkin method for the Boltzmann-Poisson equations, Boletin de la Sociedad Espanola de Matematica Aplicada, 54, 47-64 (2011)

[15] O. Muscato, W. Wagner, A Class of Stochastic Algorithms for the Wigner Equation, SIAM J. Sci. Comput., 38(3), A1483-A1507 (2016)

[16] V. D. Camiola, V. Romano, Hydrodynamical Model for Charge Transport in Graphene, J. Stat. Phys., 157, 1114-1137 (2014)

[17] G. Mascali, V. Romano, Si and GaAs mobility derived from a hydrodynamical model for semiconductors based on the maximum entropy principle, Physica A, 352, 459-476 (2005)

[18] V. Romano, Quantum corrections to the semiclassical hydrodynamical model of semiconductors based on the maximum entropy principle, J. Math. Phys., 48, 123504 (2007)

[19] V. Romano, 2D Simulation of a Silicon MESFET with a Nonparabolic Hydrodynamical Model Based on the Maximum Entropy Principle, J. Comput. Phys., 176, 70-92 (2002)

[20] S. La Rosa, V. Romano, The maximum entropy principle hydrodynamical model for holes in silicon semiconductors: the case of the warped bands, J. Phys. A: Math. Theor., 41, 215103 
(2008)

[21] S. La Rosa, G. Mascali, V. Romano, Exact Maximum Entropy Closure of the Hydrodynamical Model for Si Semiconductors: The 8-Moment Case, SIAM J. Appl. Math., 70(3), 710-734 (2009)

[22] O. Muscato, V. Di Stefano, Electrothermal Transport in Silicon Carbide Semiconductors via a Hydrodynamic Model, SIAM J. Appl. Math., 75(4), 1941-1964 (2015)

[23] G. Mascali, A New Formula for Thermal Conductivity Based on a Hierarchy of Hydrodynamical Models, J. Stat. Phys., 163(5), 1268 (2016)

[24] G. Mascali, V. Romano, Maximum entropy principle in relativistic radiation hydrodynamics, Ann. Inst. Henri Poincaré, Physique théorique, 67(2) 123-144 (1997)

[25] G. Alì, G. Mascali, V. Romano, C. R. Torcasio, A Hydrodynamical Model for Covalent Semiconductors, with Applications to GaN and SiC, Acta Appl. Math., 122(1), 335 (2012)

[26] C. Kittel, Introduction to Solid State Physics, 7th ed, Wiley India Pvt. Limited (2007)

[27] C. Jacoboni, Theory of Electron Transport in Semiconductors, Springer-Verlag (2013)

[28] F. Rana, Electron-hole generation and recombination rates for Coulomb scattering in graphene, Physical Review B, vol. 76, 155431 (2007)

[29] T. Winzer, A. Knorr, E. Malic, Carrier multiplication in graphene, Nano Letters, vol. 10, 4839-4843 (2010) 